\title{
Fluid-Measurement Technology using Flow Birefringence of Nanocellulose
}

\author{
Shogo Yamashita \\ The University of Tokyo \\ yamashi@acm.org \\ Takashi Miyaki \\ The University of Tokyo \\ miyaki@acm.org
}

\author{
Takaaki Kasuga \\ Osaka University \\ tkasuga@eco.sanken.osaka-u.ac.jp \\ Masaya Nogi \\ Osaka University \\ nogi@eco.sanken.osaka-u.ac.jp
}

\author{
Shunichi Suwa \\ The University of Tokyo \\ Shunichi.Suwa@sony.com \\ Jun Rekimoto \\ The University of Tokyo \\ rekimoto@acm.org
}

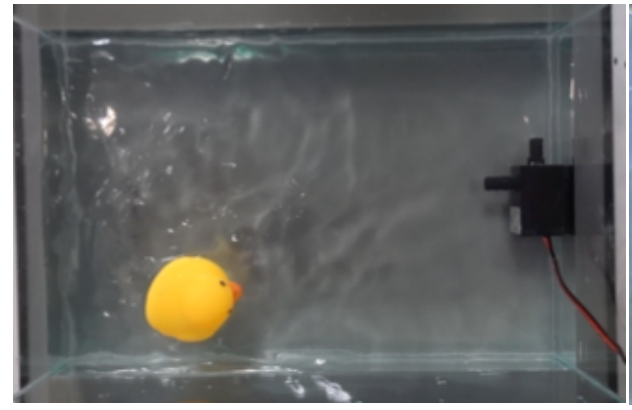

(A) A pump creating flows in a water tank

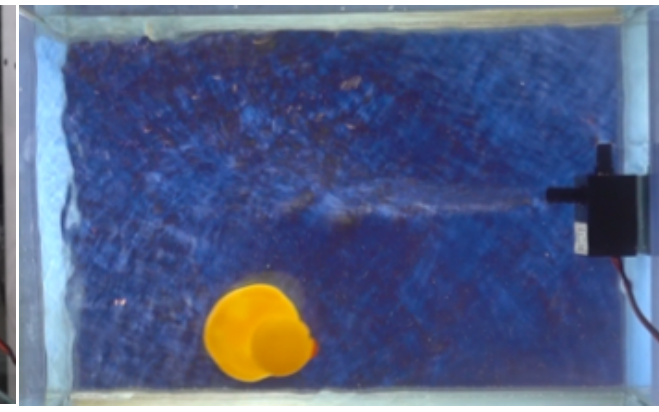

(B) Visualized water flows from a pump

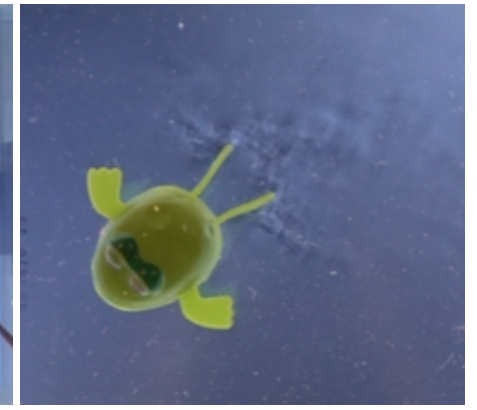

(C) Flows from a swimming toy

Figure 1: A novel fluid-measurement technology using nanocellulose. Cellulose nanofiber dispersion (3-15 nm-wide cellulose nanofibers, $0.25 \mathrm{wt} \%$ ) is filled in a water tank (A). Flows in the tank are visualized as the movement of white patterns when placed between right and left circularly polarizing plates $(B, C)$.

\begin{abstract}
We propose a potential fluid-measurement technology aimed at supporting biomechanics research of water sports using fluid simulation and motion analysis. Cellulose nanofibers introduced into the water as tracer particles to visualize the movement of water. An optical property of nanofibers, called flow birefringence, makes water flows brighter than their surroundings when placed between right and left circularly polarized plates. We tested the capability of the technology in a water tank and succeeded in using an existing particle-tracking method-particle image velocimetry (PIV)-to measure the flows from a pump in the tank.
\end{abstract}

\section{CCS CONCEPTS}

- Human-centered computing $\rightarrow$ Visualization techniques.

\section{KEYWORDS}

fluid measurement, flow Visualization, PIV, nanocellulose

Permission to make digital or hard copies of part or all of this work for personal or classroom use is granted without fee provided that copies are not made or distributed for profit or commercial advantage and that copies bear this notice and the full citation on the first page. Copyrights for third-party components of this work must be honored For all other uses, contact the owner/author(s).

SIGGRAPH '19 Posters, July 28 - August 01, 2019, Los Angeles, CA, USA

(c) 2019 Copyright held by the owner/author(s).

ACM ISBN 978-1-4503-6314-3/19/07.

https://doi.org/10.1145/3306214.3338565
ACM Reference Format:

Shogo Yamashita, Takaaki Kasuga, Shunichi Suwa, Takashi Miyaki, Masaya Nogi, and Jun Rekimoto. 2019. Fluid-Measurement Technology using Flow Birefringence of Nanocellulose. In Proceedings of SIGGRAPH '19 Posters. ACM, New York, NY, USA, 2 pages. https://doi.org/10.1145/3306214.3338565

\section{INTRODUCTION}

Fluid measurement has many significant applications in scientific and engineering research, such as evaluation of fluid simulation, design of underwater vehicles, and development of artificial hearts and engines. Fluid measurement is also an active research area in the biomechanics of water sports [Si et al. 2014; Wei et al. 2014]. Most water sports, such as swim racing, emphasize moving speed in swimming pools. According to previous studies, understanding water flow around a swimmer is key to reducing water resistance when swimming and improving propulsive force [Narita et al. 2018]. Flows are usually visualized by introducing micro-sized plastic particles, called tracer particles, into the fluid. Cameras track the movement of the particles to measure the flow. Such a measurement needs to be taken in a completely dark room, and the measurement system requires a strong laser light source illuminating the particles in the fluid [Raffel et al. 2007]. This optical system makes the tracer particles sufficiently brighter than their surroundings, thereby enabling the cameras to track the particles. For 2D flow measurements, a flatly widened laser called a laser sheet illuminates a layer of tracer particles buoyed in the fluid. However, this method 
has potential adverse effects for humans, such as laser burns and injuries from swimming in the dark. Generally, category 4 laser light sources are used for wide-field fluid measurement. Such a strong laser can cause severe eye and/or skin damage, so a less powerful light source is required to measure water flows around humans. In this research, we propose a potential fluid-measurement technology using nanocellulose as tracer particles and a harmless light source. This technology has significant contributions to biomechanics research using fluid simulation and motion analysis by providing a way to evaluate the simulation results. The technology potentially achieves the following:

- A human-friendly water flow measuring technology using harmless tracer particles and a harmless light source.

- The measurable area can be technically extended to encompass a human swimmer.

- Cellulose nanofibers are uniformly distributed in the water and do not cohere to each other, and achieve a quite slow sinking speed since the terminal speed of nano-order particles is significantly lower than existing micro-order tracer particles. This achieves uniform fluid measurements in the entire measurement field.

- An existing particle tracking method-particle image velocimetry (PIV)-can successfully be used for flow measurements.

\section{IMPLEMENTATION}

The optical setup for visualizing the flow consists of two types of circularly polarizing plates (right and left) and a white LED backlight, which is installed behind the plates (Figure 1 [A]). Tracer particles (cellulose nanofibers) are distributed in the water tank placed between the polarizing plates. Cellulose nanofiber is used in cosmetics and should be harmless to human skin. In this study, we coated the backlight with left circularly polarizing plates and put right circularly polarizing plates in front of the cameras. The combination of the circularly polarizing plates does not permit light to pass from behind. Thus, the light from the backlight is shielded, and the backlight coated with the polarization plate (left) seems dark when viewed through a right circularly polarizing plate. However, tracer particles with birefringence change the optical property and allow the light from the back to be seen through the particles. Cellulose nanofibers are fibers with a high aspect ratio. When the nanofibers are aligned in the same orientation, they show strong birefringence. The alignment of the nanofibers is achieved by flows. This birefringence result from flows is called flow birefringence [Mtibe et al. 2015]. The water flows seem brighter than their surroundings due to this phenomenon, as shown in (Figure $1[\mathrm{~B}],[\mathrm{C}]$ ).

Figure 2 shows the result of flow measurement using particle image velocimetry (PIV). PIV is a common flow visualization method that tracks the movement of patterns composed of several particles. We placed a pump in a water tank $(30 \mathrm{~cm} \times 20 \mathrm{~cm} \times 25 \mathrm{~cm})$ and measured the water flows in the tank. We put $5 \mathrm{mg}$ of cellulose nanofiber (3-15 nm-wide cellulose nanofiber sold as TEMPO-oxidized cellulose nanofiber) into $2 \mathrm{~L}$ of water as tracer particles in this test. The nanocellulose dispersion had a concentration of $0.25 \mathrm{wt} \%$. We took video of the water flows using a Sony RX10 V with camera settings as follows: exposure $=0.0 \mathrm{EV}$, F-stop $=\mathrm{f} / 2.8$, shutter speed $=1 / 500$,
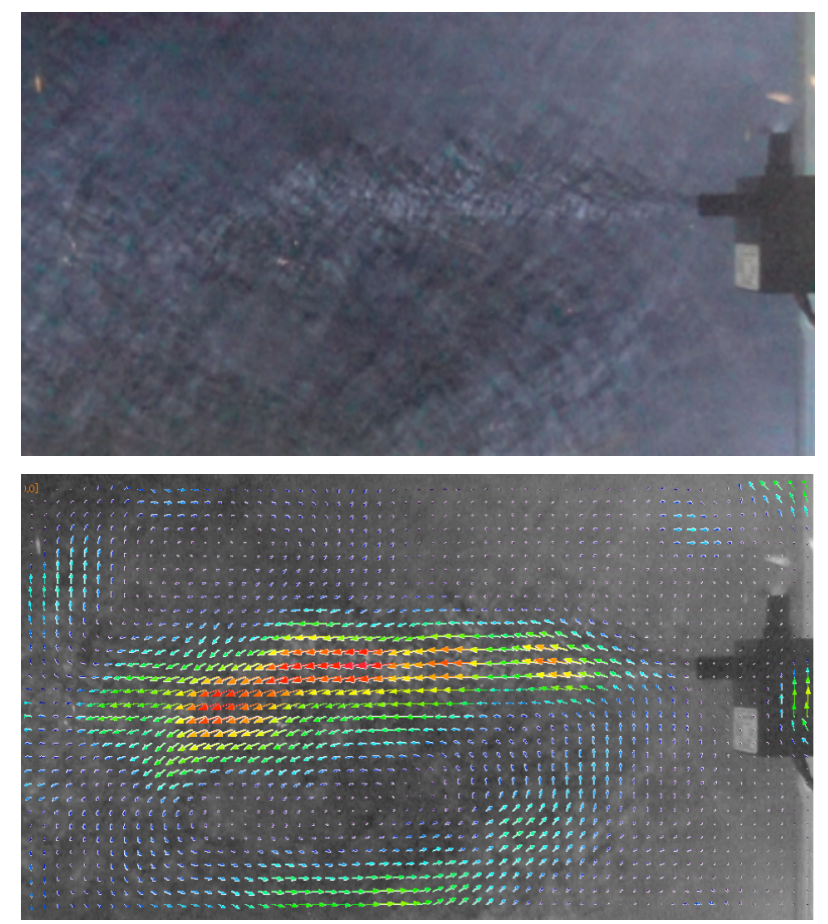

Figure 2: The result of flow measurement using a typical flow visualization method called particle image velocimetry (post processing). The arrows represent the speed and directions of flows.

and FPS $=480$. We used software called PIVview (PIVTEC GmbH, Germany) for flow measurement. Multiple cameras capturing different viewpoints from multiple axes would be able to restore the full 3-D flow using tomography.

\section{CONCLUSION AND FUTURE WORK}

We propose a fluid-measurement setup for using nanocellulose as tracer particles. This technology will contribute in particular to the study of the biomechanics of water sports using fluid simulation and motion analysis. The effects by the inculcation of nanocellulose, such as an increase in viscosity of water and a decrease in transparency of the water, still need to be investigated.

\section{REFERENCES}

A Mtibe, Linda Z Linganiso, Aji P Mathew, Kristiina Oksman, Maya J John, and Rajesh D Anandjiwala. 2015. A comparative study on properties of micro and nanopapers produced from cellulose and cellulose nanofibres. Carbohydrate Polymers 118 (2015), 1-8.

Kenzo Narita, Motomu Nakashima, and Hideki Takagi. 2018. Effect of leg kick on active drag in front-crawl swimming: comparison of whole stroke and arms-only stroke during front-crawl and the streamlined position. fournal of Biomechanics (2018).

Markus Raffel, Christian E Willert, Jürgen Kompenhans, et al. 2007. Particle image velocimetry: a practical guide. Springer Science \& Business Media.

Weiguang Si, Sung-Hee Lee, Eftychios Sifakis, and Demetri Terzopoulos. 2014. Realistic biomechanical simulation and control of human swimming. ACM Transactions on Graphics (TOG) 34, 1 (2014), 10.

Timothy Wei, Russell Mark, and Sean Hutchison. 2014. The fluid dynamics of competitive swimming. Annual Review of Fluid Mechanics 46 (2014), 547-565. 